\title{
Unusual Complex Coacervation between Neutral Polymer and Polyoxometalate in Aqueous Solution via Direct Water Mediation
}

Benxin Jing a, ${ }^{\mathrm{a}}$, Manuela Ferreira ${ }^{\mathrm{a}}$, Yunyi Gao ${ }^{\mathrm{b}}$, Christopher Wood ${ }^{\mathrm{a}}$, Ruipeng $\mathrm{Li}^{\mathrm{c}}$, Masafumi Fukuto $^{\mathrm{c}}$, Tianbo Liu ${ }^{\mathrm{b}}$, and Yingxi Zhu,

${ }^{a}$ Department of Chemical Engineering and Materials Science, Wayne State University, Detroit, MI 48202, USA

${ }^{\mathrm{b}}$ Department of Polymer Science, The University of Akron, Akron, OH 44325, USA

${ }^{\mathrm{c}}$ National Synchrotron Light Source II, Brookhaven National Laboratory, Upton, NY 11973, USA

*Corresponding authors (Email: jingbenxin@gmail.com (B. Jing), yzhu3@wayne.edu (Y. Zhu))

\section{Supporting Information}

Generality of PEG-POM complex coacervation with different POMs. The generality of PEGPOM coacervate formation was confirmed with other POMs of similar atomic architectural structure but varied chemical composition and size, including silicotungstic acid $\left(\mathrm{H}_{4} \mathrm{SiW}_{12} \mathrm{O}_{40}\right.$, $\left.\left\{\mathrm{SiW}_{12}\right\}\right)$, and phosphomolybdic acid $\left(\mathrm{H}_{3} \mathrm{PMo}_{12} \mathrm{O}_{40},\left\{\mathrm{PMo}_{12}\right\}\right)$. Similar liquid-liquid separated PEG-POM complex coacervation is observed with these Keggin POMs as shown in Figure S1. $\left\{\mathrm{SiW}_{12}\right\}$ and $\left\{\mathrm{PMo}_{12}\right\}$ were purchased from Sigma-Aldrich and used directly.

Figure S1 (a) Digital photographs of PEG-100k complex coacervates formed with (i) $\left\{\mathrm{SiW}_{12}\right\}$ and (ii) $\left\{\mathrm{PMo}_{12}\right\}$ in $\mathrm{LiCl}$ added aqueous solution of $c_{E G}^{i}=1.135 \mathrm{M}$ and $c_{L i C l}=2.0 \mathrm{M}$. (b) Fluorescence micrographs of (i) the supernatant and (ii) the dense phase of PEG-100k complex coacervates formed with $\left\{\mathrm{SiW}_{12}\right\}$, for which FITC fluorophore is added to the aqueous solutions before mixing. The micrographs were acquired by CLSM with an Airy detector. The scale bar for both fluorescence micrographs is $20 \mu \mathrm{m}$. 
(a)
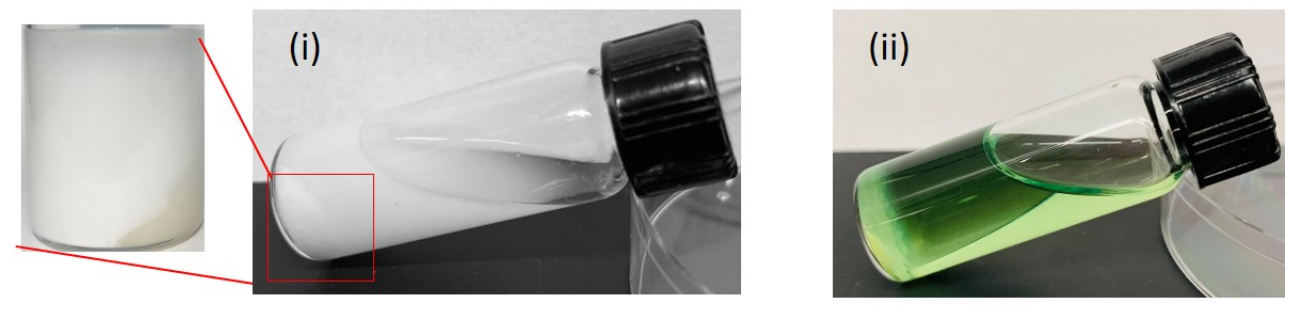

(b)
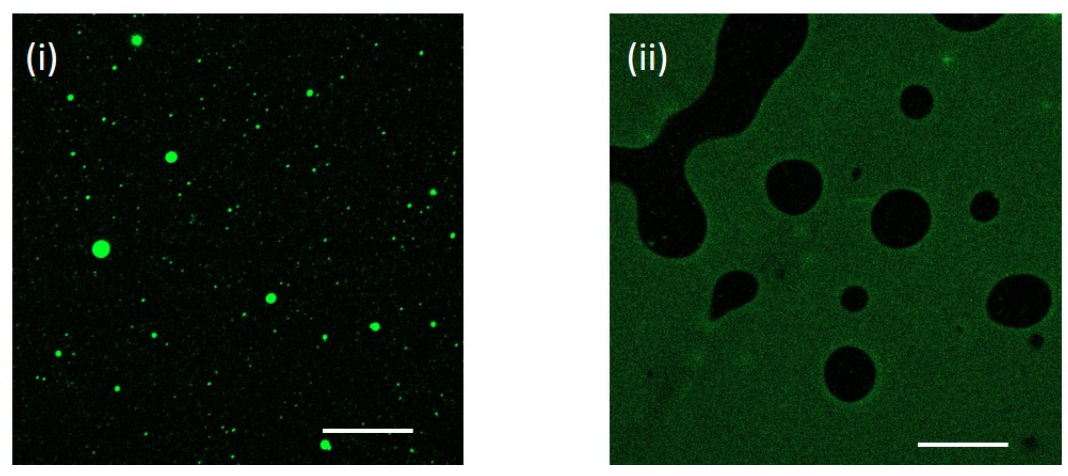

Effects of salt concentration on PEG-POM coacervate formation. We have observed that increasing $\mathrm{LiCl}$ concentration in aqueous solution before mixing could lower $c_{\{W 12\}}^{c}$, suggesting the promotion of $\mathrm{LiCl}$ for PEG-POM coacervate formation in contrast to salt-induced instability and re-dissolution of polyelectrolyte complex coacervates.

Figure S2 Effect of $\mathrm{LiCl}$ concentration on $\mathrm{c}_{\{W 12\}}^{c}$ for the onset of PEG- $\left\{\mathrm{W}_{12}\right\}$ coacervate complex formation at constant initial $c_{E G}^{i}=1.135 \mathrm{M}$ for PEG-4k (black squares), PEG-10k (red circles), and PEG-100k (blue triangles).

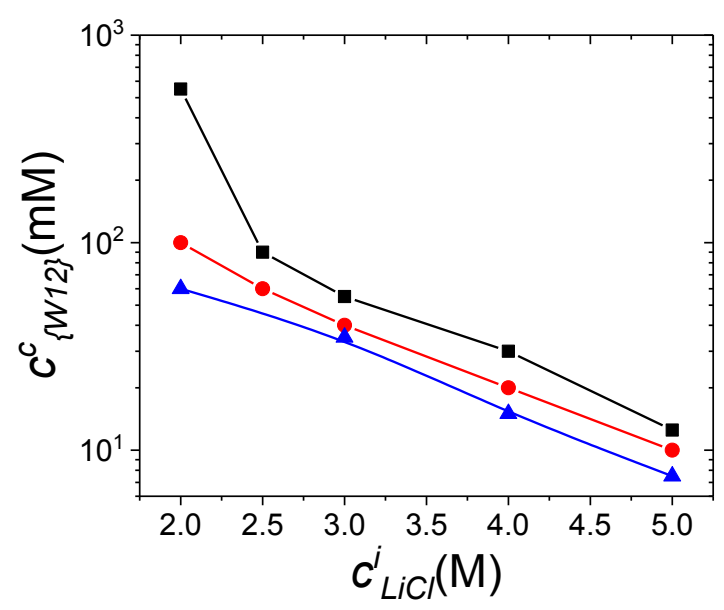


Figure S3 (a) Guinier plot of $I(q)$ against $q^{2}$, (b) Porod plot of $I(q) * q^{4}$ against $q$, and (c) PorodDebye plot of $I(q) * q^{4}$ against $q^{4}$ for the cases as shown in Figure 3.

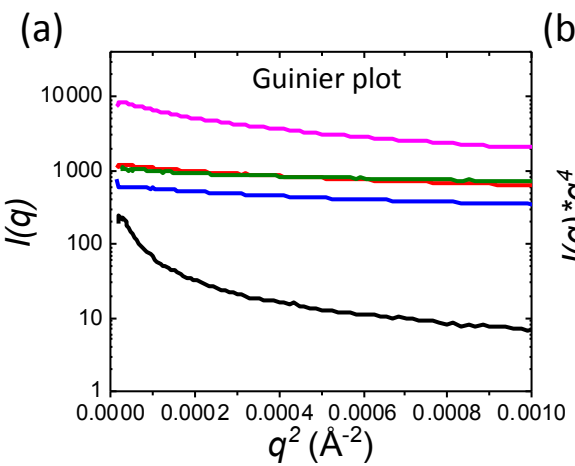

(b)
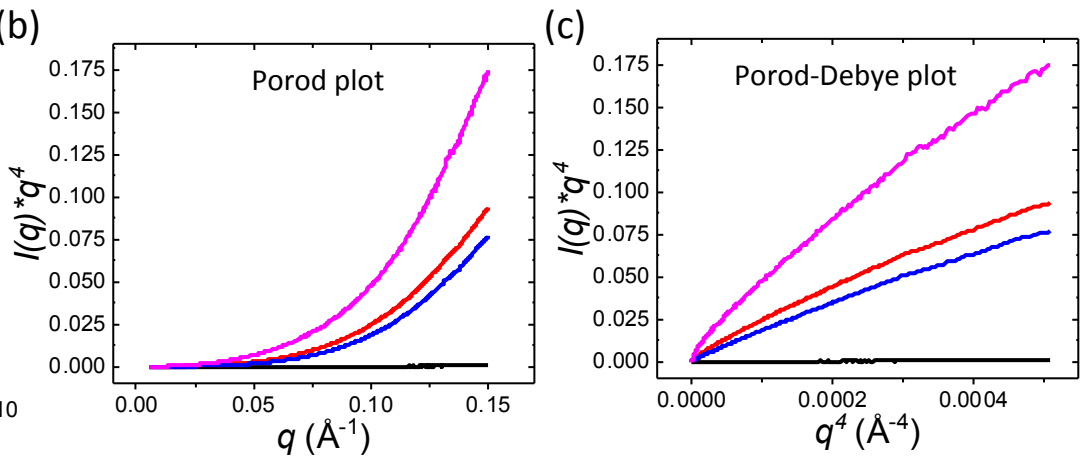

Microscopic structure of PEG- $\left\{W_{12}\right\}$ complex coacervates. The loose nanostructure of PEG$\left\{\mathrm{W}_{12}\right\}$ coacervate phase is also characterized by fluorescence recovery after photobleaching (FRAP) using CLSM. Fluorescent f-PEG was used as the fluorescence probe and mixed with PEG aqueous solutions at f-PEG:PEG weight ratio of 1:9 in PEG-LiCl aqueous solution before its mixing with $\left\{\mathrm{W}_{12}\right\}$ solution. Measured $F(t)$ over the bleached circular area of $d=32 \mu \mathrm{m}$ in diameter, normalized by that of the unbleached region of the same diameter, $F(0)$, was plotted against lapsed time, $t$ as shown in Figure S4b. The fluorescence intensity of added f-PEG could be $100 \%$ recovered after photobleaching, indicating highly mobile PEG in complexes.

Figure S4 (a) Representative fluorescence micrographs exhibit the fluorescence recovery after photobleaching of PEG- $\left\{\mathrm{W}_{12}\right\}$ coacervate phase formed at $c_{E G}^{i}=1.135 \mathrm{M}, c_{\{W 12\}}^{i}=100 \mathrm{mM}$ and $c_{\text {LiCl }}=2.0 \mathrm{M}$. One circular area (in the lower right corner) of diameter, $d=32 \mu \mathrm{m}$ was bleached and recorded in comparison to one unbleached circular area (in the upper left corner) of the same diameter as the reference. The rest of the area shown in the micrographs was not scanned to reduce the damaging of laser on fluorescence dye. (b) Measured fluorescence intensity, $F(t)$ (black circles) over the bleached circular area of $d=32 \mu \mathrm{m}$ in diameter as shown in (a), normalized by that of the unbleached region of the same diameter, $F(0)$, against time. Red solid line shows the exponential fitting of measured $F(t) / F(0)$ against time. 
(a)
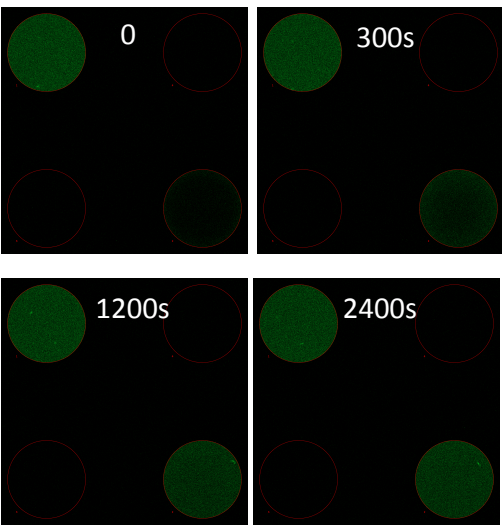

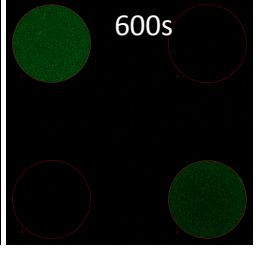

4800 s

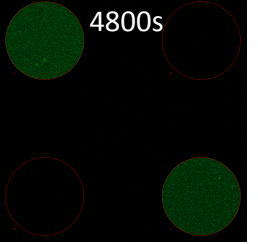

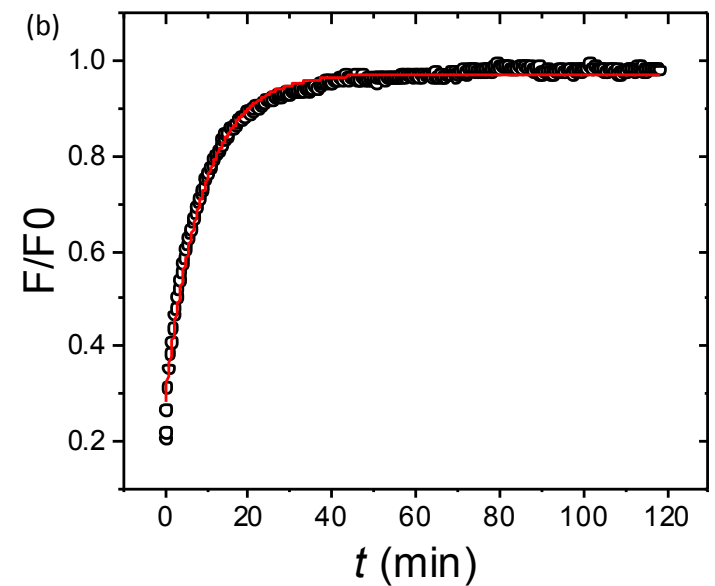

Figure S5 Titration curve of $\left\{\mathrm{W}_{12}\right\}$ by adding $0.2 \mathrm{M} \mathrm{NaOH}$ solution to $100 \mathrm{~g} / \mathrm{L}\left\{\mathrm{W}_{12}\right\}$ solution (black squares). The transition data at $\mathrm{pH}>8$ indicates the decomposition of $\left\{\mathrm{W}_{12}\right\}$ nanoclusters in basic aqueous solution. As a control, the titration curve of adding $\mathrm{NaOH}$ solution to $0.1 \mathrm{M} \mathrm{HCl}$ solution is included (red circles).

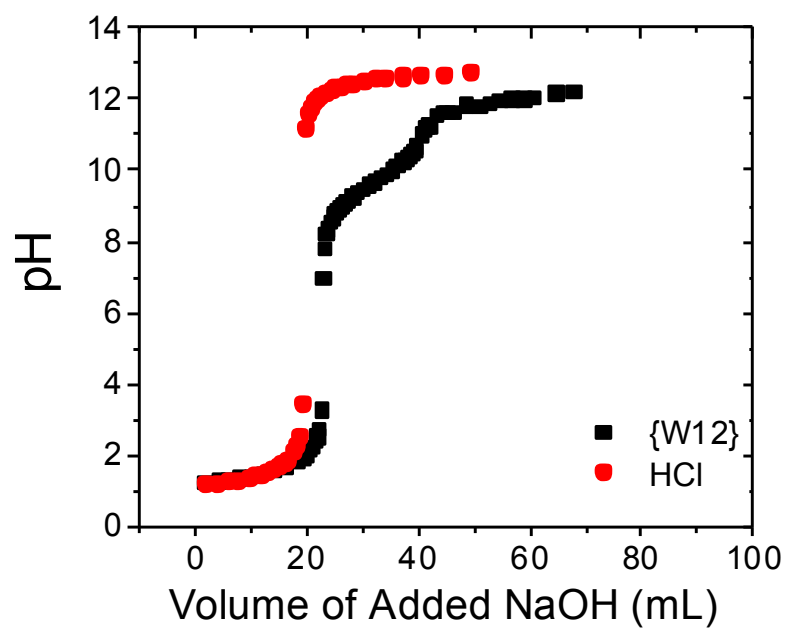


Figure S6 Thermogravimetric analysis (TGA) of freeze-dried $\left\{\mathrm{W}_{12}\right\}$ at a heating rate of $10{ }^{\circ} \mathrm{C}$ $\min ^{-1}$ with $\mathrm{N}_{2}$ purge of $60 \mathrm{~mL} \mathrm{~min}^{-1}$. TGA measurement was carried out by using TGA Q50 (TA Instruments).

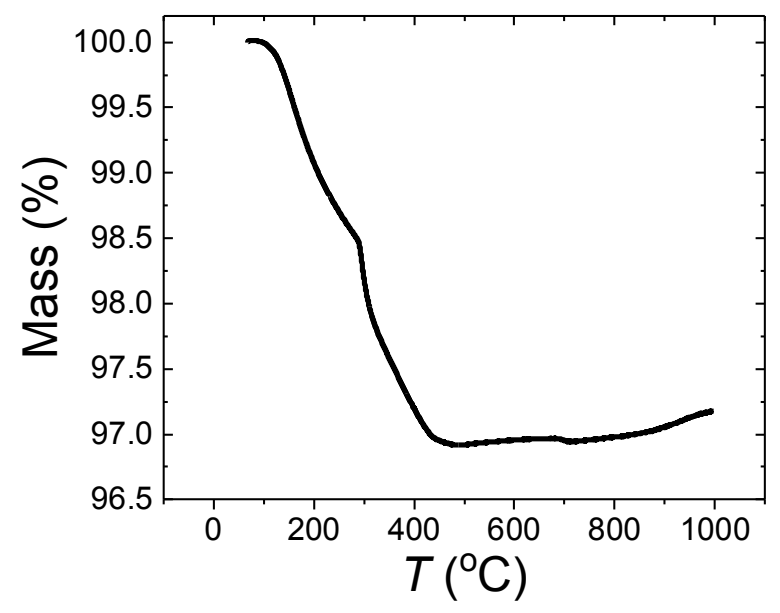

